\title{
An attempt to optimize the outcome of penetrating keratoplasty in congenital aniridia-associated keratopathy (AAK)
}

\author{
C. J. Farah $(\mathbb{D} \cdot$ F. N. Fries $・$ L. Latta $・$ B. Käsmann-Kellner • B. Seitz
}

Received: 9 February 2021 / Accepted: 16 July 2021 / Published online: 29 July 2021

(C) The Author(s) 2021

\begin{abstract}
Purpose To propose an optimized microsurgical and medical approach to reduce the risk of complications after penetrating keratoplasty (PKP) in patients with aniridia-associated keratopathy (AAK).

Methods Retrospective observational case series of 25 PKP performed in 16 patients with AAK. Preoperative indications were endothelial decompensation and vascularized scars $(68 \%)$ or graft failure $(32 \%)$ due to limbal stem cell deficiency. The optimized approach included a combination of a small corneal graft size (around $7.0 \mathrm{~mm}$ ), interrupted 10-0nylon sutures, simultaneous AMT as a patch, large bandage contact lens, temporary lateral tarsorrhaphy, postoperative autologous serum eye drops, and systemic immunosuppression. Main outcome measures included: visual acuity, transplant survival, and complications encountered during follow-up of 107 weeks on average.
\end{abstract}

C. J. Farah $(\bowtie)$ · F. N. Fries · B. Käsmann-Kellner ·

B. Seitz

Department of Ophthalmology, Saarland University

Medical Center, 100 Kirrbergerstr., Building 22,

66421 Homburg, Saar, Germany

e-mail: farah.chr.j@gmail.com

L. Latta

Dr. Rolf M. Schwiete Center for Limbal Stem Cell

Research and Congenital Aniridia, Saarland University

Medical Center, Homburg, Saar, Germany
Results A complete modified keratoplasty scheme was used in 10 of 25 PKP (group 1), while at least one of the modifications was missing in the other 15 PKP (group 2). After 8 weeks of follow-up, the epithelium was closed in 23 eyes. Visual acuity improved in 19 eyes at 6 months of follow-up, and remained stable in six eyes. None of the eyes showed a decrease in visual acuity. At the last post-operative follow-up, this visual improvement persisted in 14 eyes and graft survival rate after 156 weeks ( 3 years) was $69 \%$ in group 1 versus $44 \%$ in group 2 ( $p=0.39$, log-rank test). Secondary corneal neovascularization $(8 \%)$, scarring $(4 \%)$, ulcer $(4 \%)$, or graft rejection $(8 \%)$ happened mostly in the second group which was missing at least one of the suggested modifications.

Conclusions PKP in congenital aniridia must be considered as a high-risk keratoplasty. An optimized therapeutic approach seems to be promising in order to reduce the postoperative complication rate in these most difficult eyes.

Keywords Aniridia-associated keratopathy . Penetrating keratoplasty · Limbal stem cell deficiency - Amnion membrane transplantation . Autologous serum 


\section{Introduction}

First described as "congenital irideremia" in the nineteenth century, congenital aniridia is a rare (1:60.000-1:90.000) pan-ocular disease that can be differentiated in two major categories depending on the presence of a PAX-6-Gene mutation [1-4]. Among those with a mutation of the PAX-6-Gene, frequent mutations involve point mutations and deletions. A deletion of the short arm of chromosome 11(p13) may be autosomal dominant or sporadic, and to a lesser extent autosomal recessive, e.g., in the Gillespie syndrome [5]. A PAX-6-Gene mutation is more frequently associated with ocular complications compared to aniridia triggered by other mutations than the PAX-6-Gene [2, 3]. Congenital aniridia is linked to different malformations such as iris, macular or optic nerve hypoplasia, but also to deteriorating progressive major ocular dysfunctions such as limbal stem cell deficiency, premature onset of cataract, and secondary glaucoma that can lead to blindness throughout life [2, 3, 6-8]. The incidence and severity of aniridiaassociated keratopathy (AAK) increases with age affecting about $20-30 \%$ of those patients and leads to corneal opacities, scarring, and vascularization due to a unique form of limbal stem cell deficiency [2,9-11].

With time, it may result in corneal ulcers, dense vascularized scars, or endothelial decompensation (especially after complicated cataract and/or glaucoma surgery), where a penetrating keratoplasty becomes indicated [8]. Persisting epithelial defects, suture loosening, and an increased risk of graft rejection are typical postoperative complications in those high-risk keratoplasties [8]. We hypothesized that the combination of a small corneal graft size, interrupted sutures, simultaneous amniotic membrane transplantation (AMT) as a patch [12, 13], large bandage contact lens, temporary lateral tarsorrhaphy, postoperative autologous serum eye drops [14], and systemic immunosuppression $[15,16]$ may improve the outcome after PKP in congenital aniridia.

\section{Patients and methods}

This study is a retrospective observational case series of 25 penetrating keratoplasties (PKP) performed in 20 eyes of 16 patients with AAK at the Department of Ophthalmology of the Saarland University Medical
Center in Germany between 2012 and 2019. Four patients received a bilateral surgery. All procedures were performed in accordance with the ethical standards of the 1964 Helsinki Declaration and its later amendments. Transplantation had been proposed as a last resort therapy in corneas with severe endothelial decompensation and stromal scars $(68 \%)$ and graft failure (32\%), due to limbal stem cell deficiency with no success of conservative treatments (stage IV or V according to Yazdanpanah et al. [17]).

The mean age during keratoplasty was $52 \pm 8$ (from 26 to 64) years, and most eyes (92\%) had a history of previous surgeries (Table 1). We defined a small graft as ranging from 6.0 to $7.5 \mathrm{~mm}$ diameter. Two types of 10-0 nylon sutures were used as follows: double-running cross-stitch sutures according to Hoffmann versus 24-32 interrupted sutures (Fig. 1). Amniotic membranes were collected from healthy women with their consent and properly processed in the eye bank before transplantation [18]. They were transplanted at the end of the keratoplasty as a single 16-mm layer membrane with the stromal side facing the corneal graft and fixed with a running 10-0 nylon episcleral suture as a patch [12]. The membrane was covered with a large 17-mm bandage contact lens, and both sutures and contact lens were removed after a period of 4-6 weeks. Temporary lateral tarsorrhaphy was performed using 5-0 silk and left typically for 4-6 weeks after PKP.

After exclusion of systemic infectious diseases, $100 \%$-concentrated autologous serum eye drops were prepared $[19,20]$. During the first postoperative days, they were applied hourly alternating with preservative-free hyaluronic acid containing artificial tear eye drops. A topical antibiotic coverage for at least 4 weeks with ofloxacin or moxifloxacin eyedrops five times daily was necessary to prevent infections until complete epithelial healing was achieved, and the bandage contact lens was removed, typically after 4 weeks. An additional long-term therapy with topical acyclovir (five times daily) and systemic acyclovir (starting with $400 \mathrm{mg}$ five times daily for 6 weeks, then two times daily for 1 year) was given to patients with history of herpetic keratitis.

Postoperative topical corticosteroids (starting at five times daily, being reduced by 1 drop every 6-8 weeks) and systemic corticosteroid (prednisolone, prednisolonacetat, and methylprednisolone) were slowly tapered over 4 weeks, starting at $100 \mathrm{mg}$ 
Table 1 Characteristics of 16 patients with congenital aniridia (25 keratoplasties)

\begin{tabular}{|c|c|}
\hline Patients & $n=16(100 \%)$ \\
\hline Males & $6(37,5 \%)$ \\
\hline Females & $10(62,5 \%)$ \\
\hline Genetics & $n=16(100 \%)$ \\
\hline PAX-6 & $7(43,75 \%)$ \\
\hline WAGR(O) & $1(6,25 \%)$ \\
\hline Genetically not analyzed & $8(50 \%)$ \\
\hline Type of Keratoplasty & $n=25(100 \%)$ \\
\hline First keratoplasty (with or without simultaneous pannus removal) & $13(52 \%)$ \\
\hline Repeat keratoplasty (with or without simultaneous pannus removal) & $5(20 \%)$ \\
\hline Classical triple procedure (combined with cataract surgery and intraocular lens implantation) & $4(16 \%)$ \\
\hline Pole-to-pole surgery (keratoplasty combined with lens and vitreoretinal surgery) & $2(8 \%)$ \\
\hline HLA-typed keratoplasty & $1(4 \%)$ \\
\hline History of previous surgeries before keratoplasty (Multiple choices possible) & $n=25(100 \%)$ \\
\hline Glaucoma surgery & $11(44 \%)$ \\
\hline Trabeculotomy & $3(12 \%)$ \\
\hline Cyclophotocoagulation & $9(36 \%)$ \\
\hline Ahmed valve & $5(20 \%)$ \\
\hline Corneal surgery & $12(48 \%)$ \\
\hline Pannus removal (“pannectomy”) & $3(12 \%)$ \\
\hline Phototherapeutic keratectomy (PTK) & $1(4 \%)$ \\
\hline Penetrating keratoplasty (in domo) & $5(20 \%)$ \\
\hline Penetrating keratoplasty (ex domo) & $3(12 \%)$ \\
\hline Cataract surgery & $8(32 \%)$ \\
\hline Retinal surgery & $4(24 \%)$ \\
\hline None & $2(8 \%)$ \\
\hline
\end{tabular}

daily and being reduced by $20 \mathrm{mg}$ every second day until $20 \mathrm{mg}$ daily, then being reduced slowly. A systematic preoperative evaluation for long-term use of immunosuppression with mycophenolate mofetil or cyclosporin A and systemic follow-up were established in collaboration with the family physician, and doses were adapted to the general condition of the patient.

Transplantations were combined with simultaneous cataract or vitreoretinal surgeries where indicated. Characteristics of recipients are shown in Table 1 . The optimized approach included a combination of a small corneal graft size (around $7.0 \mathrm{~mm}$ ), interrupted 10-0 nylon sutures, simultaneous AMT as a patch, large bandage contact lens, temporary lateral tarsorrhaphy, postoperative autologous serum eye drops, and systemic immunosuppression. Main outcome measures included: visual acuity, transplant survival, and complications encountered during the follow-up of 107 weeks on average.

For statistical analysis, eyes were separated into two groups based on the therapeutic scheme. A complete, modified keratoplasty scheme was used in 10 of 25 PKP (group 1). The second group included all other PKP, where at least one modification of the modified keratoplasty scheme was missing (group 2). Techniques used are illustrated in Table 2.

Statistical analysis was performed using SPSS v. 20.0.0 (IBM Corp., Armonk, NY, USA). Visual acuity was recorded in decimal and converted into logMAR before analysis. Graft survival was analyzed using the Kaplan-Meier method and log-rank test, which is appropriate to compare small sample sizes and unequal censoring groups. 

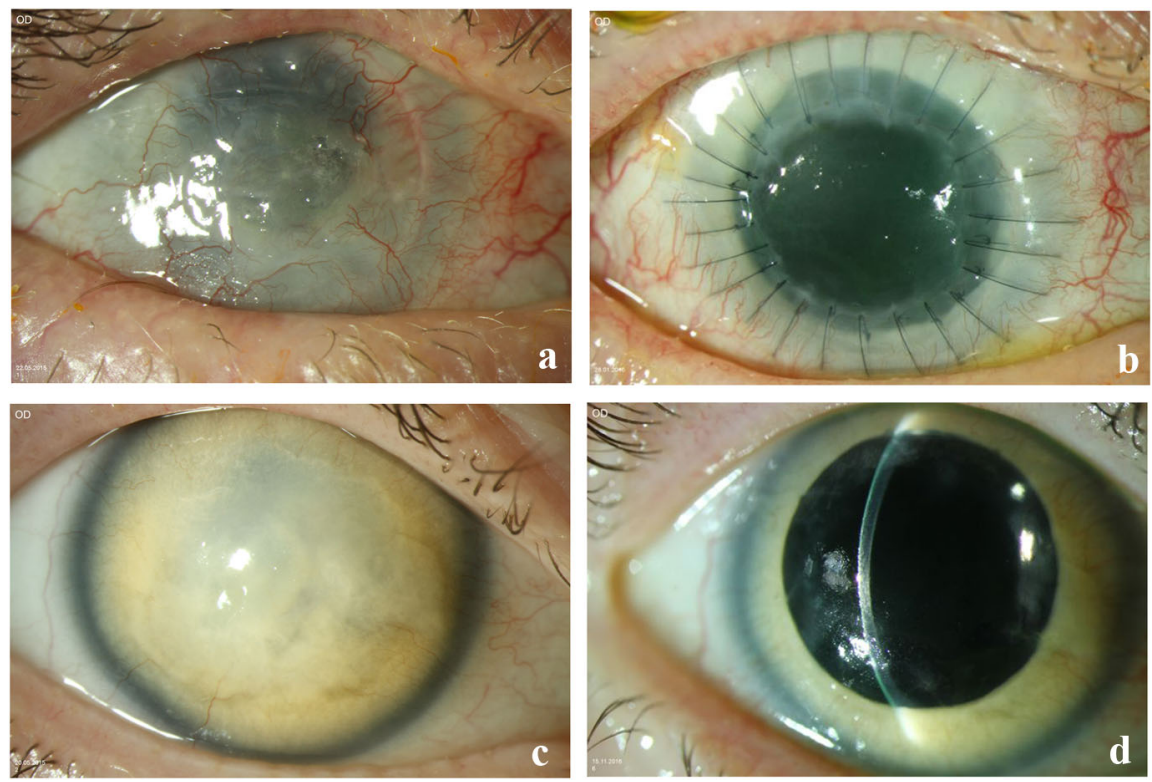

Fig. 1 a Patient 1: Preoperative picture with decompensated

64-year old woman with corneal decompensation, vascularized and severely vascularized graft, 6 years after penetrating keratoplasty. Visual acuity: Hand motion (logMar 2.7). b Patient 1: 8 months postoperative picture with 26 interrupted sutures after repeat penetrating keratoplasty with Barron trephine (7.00/ $7.25 \mathrm{~mm}$ ). Visual acuity has reached $\log$ Mar 2.3. c Patient 2: cornea, secondary amyloidosis, and premature cataract in congenital aniridia. Visual acuity: Hand motion (logMAR 2.7). d Patient 2: Clear corneal graft, 18 months after classical excimer laser-assisted triple procedure $(7.0 / 7.1 \mathrm{~mm})$ and suture removal. Visual acuity improved to $\log$ MAR 1.0

Table 2 Techniques used in 25 keratoplasties (Multiple choices possible)

\begin{tabular}{ll}
\hline Diameter of recipient openings (diameter in mm) & $n=25(100 \%)$ \\
\hline $6.5 \mathrm{~mm}$ & $1(4 \%)$ \\
$7.0 \mathrm{~mm}$ & $14(56 \%)$ \\
$7.5 \mathrm{~mm}$ & $9(36 \%)$ \\
$12.0 \mathrm{~mm}$ (Limbo-keratoplasty) & $1(4 \%)$ \\
Sutures & $n=25(100 \%)$ \\
Interrupted sutures & $18(72 \%)$ \\
Double-running cross-stitch sutures according to Hoffmann $(1976)$ & $7(28 \%)$ \\
Use of simultaneous amnion membrane transplantation (Patch) & $n=25(100 \%)$ \\
Yes & $24(96 \%)$ \\
No & $1(4 \%)$ \\
Use of simultaneous lateral tarsorrhaphy & $n=25(100 \%)$ \\
Yes & $14(56 \%)$ \\
No & $11(44 \%)$ \\
Use of postoperative autologous serum eye drops primarily & $n=25(100 \%)$ \\
Yes & $19(76 \%)$ \\
No & $6(24 \%)$ \\
Use of long-term immunosuppressive therapy & $n=25(100 \%)$ \\
Mycophenolate mofetil & $20(80 \%)$ \\
Cyclosporin-A & $3(12 \%)$ \\
None & $2(8 \%)$ \\
\end{tabular}




\section{Results}

Visual acuity

At 6 months of follow-up, the mean visual acuity (VA) improved from logMAR 2.18 to $\log$ MAR 1.65 . None of the eyes showed a decreased VA. On the other hand, the VA remained unchanged in six eyes and improved in 19 eyes. The last examination was made on average 107 weeks postoperatively with a mean VA of $\log$ MAR 1.69 in group 1 and $\log$ MAR 1.77 in group 2.

All patients reported a subjective improvement in their visual acuity, either by increased clarity of the image, or by decreased visual discomfort caused by corneal optical phenomena such as glare. However, no specific questionnaires have been used to standardize subjective assessment of patients. This visual improvement persisted in 14 eyes until the end of follow-up (107 weeks on average).

\section{Graft survival}

At 8 weeks of follow-up, the epithelium was closed in 23 eyes and only 2 eyes needed a second AMT. Graft survival was referred to as a clear and transparent graft, without endothelial decompensation scars or corneal opacities. Graft survival is demonstrated in a Kaplan-Meier chart (Fig. 2). The mean postoperative follow-up was 119 weeks in group 1 and 216 weeks in group 2. Graft survival rate after 156 weeks (3 years) was $69 \%$ in group 1 and $44 \%$ in group $2(p=0.39, \log$ rank test). The median graft survival time was 97 weeks in group 1 and 81 weeks in group 2.

\section{Adverse events}

While only two eyes needed a repeat keratoplasty in group 1 and 3 eyes in group 2, severe corneal complications such as graft rejection $(8 \%)$, anterior segment fibrosis syndrome (4\%) or graft ulcer (16\%), mostly occurred in group 2 . Other, less severe corneal complications such as persistent epithelial defects $(20 \%)$ and premature suture removal/replacement $(28 \%)$ were present in both groups (Table 3 ).

A second AMT as patch was used to treat persistent epithelial defects in five eyes. Loose corneal sutures were removed as quickly as possible to prevent infiltrates and infections. Graft rejections were primarily treated with topical intracameral and systemic steroids, followed by a repeat keratoplasty, if needed. The two eyes that required a repeat keratoplasty after graft rejection had contraindications to systemic immunosuppressive therapy.

Extracorneal complications, regardless of the chosen therapeutic scheme, were changes in ocular pressure (hypo- and hypertension) (20\%), retinal detachment (4\%), retinal vein occlusion (4\%), and intraocular lens luxation (4\%). Those complications were handled according to the respective German guidelines.

\section{Discussion}

The development of microsurgical techniques and the knowledge of limbal stem cell function has led to a considerable improvement in the treatment and visual prognosis of eyes with congenital aniridia over the years [3-9, 21-23]. Each small improvement is helpful for those progressively visually impaired patients. The global therapeutic approach should always consider a high risk of concomitant glaucomatous damage with irreversible optic atrophy due to a mispositioning of the ciliary body processes toward the iris stump and a very short ciliary body [2, 7, 24].

In well-selected cases, the treatment of AAK with PKP has shown to be beneficial, even though the postoperative complications turned out to be more frequent in those high-risk eyes $[6,8,25]$. Our modified treatment scheme appears to reduce the severe postoperative complications and improve visual prognosis as well as graft survival in the mid-term follow-up.

At first glance, the Kaplan-Meier curve may suggest a different trend between the groups, but the log-rank test could not detect a statistically significant difference between the two groups in terms of graft survival $(p=0.39)$. We attribute this to the low number of cases, which is due to the rarity of the disease. Based on our clinical experience, we refrain from randomizing patients as we do not want to deprive them of what we believe to be the optimal treatment nowadays.

We explain this difference by the following preventive measures: the use of interrupted sutures allows a quick removal of loose sutures at the slit lamp without risking graft slippage, and thus reduces the risk of infections and secondary immune reactions 
Fig. 2 Survival of corneal grafts in group 1 (complete treatment) versus group 2 (incomplete treatment). Graft survival rate after 156 weeks (3 years) was $69 \%$ in group 1 and $44 \%$ in group $2,(p=0.39$, logrank test)

Table 3 Corneal complications during follow-up. $n=25$ keratoplasties (100\%). Group 1: Complete therapeutic modified scheme $(n=10)$. Group 2: Incomplete therapeutic scheme $(n=15)$

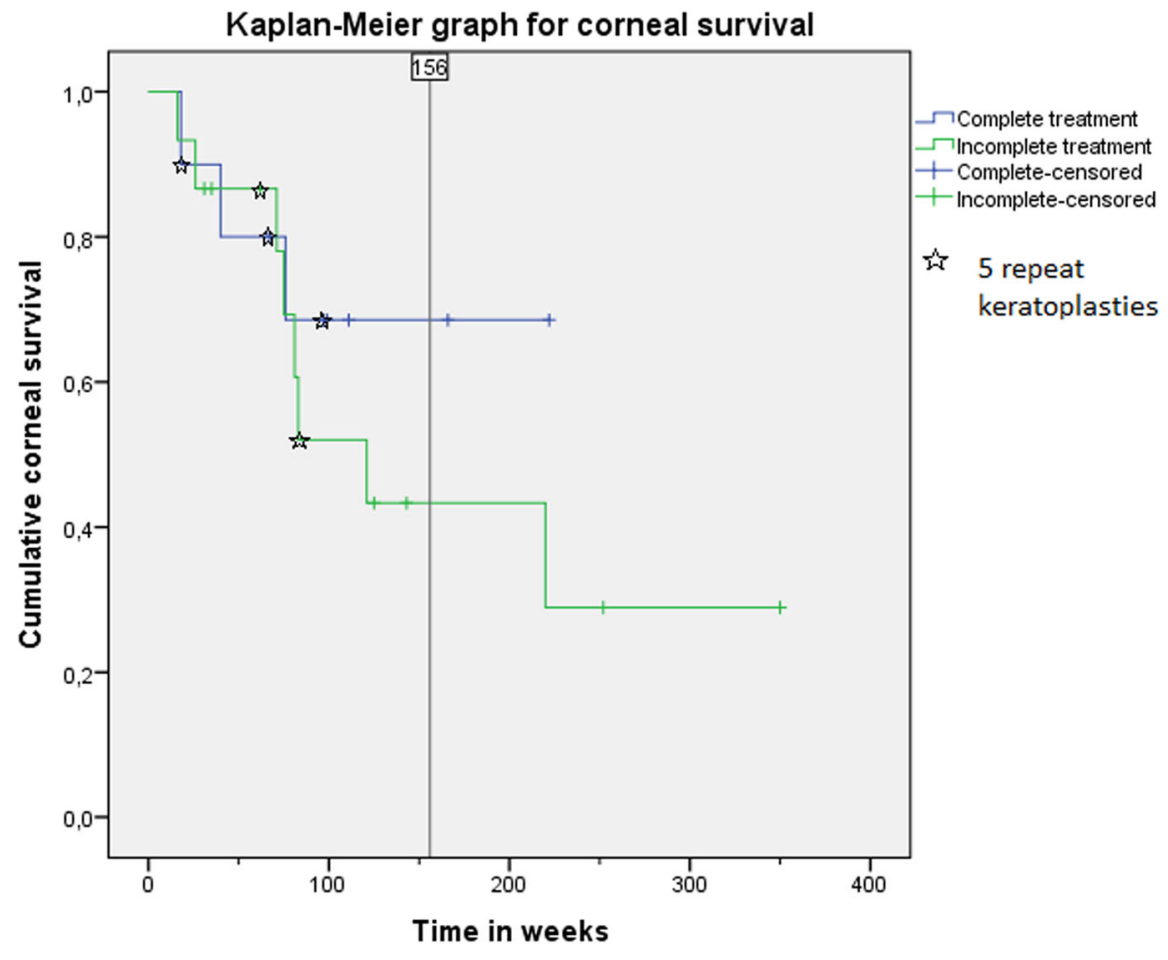

\begin{tabular}{ll}
\hline Persistent epithelial defect & $n=5(20 \%)$ \\
\hline Group 1 & $3(12 \%)$ \\
Group 2 & $2(8 \%)$ \\
Premature suture removal/replacement & $n=7(28 \%)$ \\
Group 1 & $3(12 \%)$ \\
Group 2 & $4(16 \%)$ \\
Corneal endothelial decompensation & $n=4(16 \%)$ \\
Group 1 & $2(8 \%)$ \\
Group 2 & $2(8 \%)$ \\
Immunological graft rejection & $n=2(8 \%)$ \\
Group 1 & $0(0 \%)$ \\
Group 2 & $2(8 \%)$ \\
Graft ulcer/neovascularization/scarring/anterior segment fibrosis syndrome & $n=5(20 \%)$ \\
Group 1 & $1(4 \%)$ \\
Group 2 & $4(16 \%)$ \\
\hline
\end{tabular}

[27]. A temporary lateral tarsorrhaphy combined with simultaneous AMT as a patch and 17-mm bandage contact lens considerably reduces postoperative epithelial defects due to a mechanical protective effect [12, 28, 29].

Furthermore, the amnion membrane supports epithelialization, it has anti-fibrotic effects (due to a reduced expression of TGF $\beta 1, \beta 2, \beta 3$ isoforms and
TGF-beta receptor II), and anti-inflammatory effects (through inhibition of proinflammatory cytokines). Moreover, anti-angiogenic effects (due to production of thrombospondin-1, endostatin and tissue inhibitors of metalloproteases), and immunomodulatory effects have been reported $[12,13,18,26]$. Similar positive effects have been described for the autologous serum eye drops $[13,18,25]$. Due to the higher risk of graft 
rejection, a systemic immunosuppressive therapy proved to be useful in most patients $[15,16]$.

The limitations of the study are the small sample size due to the rare condition and the lack of long-term observation periods to demonstrate a significant difference between groups. A prospective randomized study is not possible for ethical reasons. As our proposed therapeutic scheme does not directly address the cause of AAK, it could be combined with a prior transplantation of allogenic limbal stem cells, even though limbal stem cell transplantation alone in patient with AAK has been significantly associated with progression of limbal stem cell deficiency severity and visual impairment [30]. However, the survival curve looks promising, with a major separation after only 2 years of follow-up. We may expect an average graft survival of $50 \%$ at 5 years for the group which was treated with the complete scheme, and only $30 \%$ for the other group (Fig. 2). This highlights the difficulty of surgical management in AAK compared to other corneal pathologies with limbal stem cell deficiency such as herpes keratitis (survival rate of $90 \%$ after 2 years and $49 \%$ after 10 years), while chemical burn-associated limbal stem cell deficiency could best benefit from our modified scheme to enhance graft survival (survival rate of $35 \%$ after 5 years and $14 \%$ after 10 years) [31, 32]. Moreover, the four patients that received a bilateral surgery showed a better graft survival in the eyes who benefited from the optimized treatment scheme. This may also speak in favor of the efficacy of our modified scheme.

Although the Boston keratoprosthesis shows promising results in small series of patients with AAK, other complications such as retroprosthetic membrane formation or device extrusion exists $[6,33]$. Regarding the limbo-keratoplasty, Lang et al. reported a median graft survival of 3.2 years over a long-term observation, indicating that this might also be a favour of approach in "partial" limbal stem cell deficiency [34].

In conclusion, an optimized high-risk PKP approach seems to be promising to reduce the postoperative complication rate in these most difficult eyes with congenital aniridia. Furthermore, an extension to treat other high-risk corneal pathologies with limbal stem cell deficiency seems promising (e.g., chemical burn).
Author contribution CJF collected data, conceived, and designed the analysis and wrote the paper. FNF performed analysis and collected data. LL contributed data or analysis tools. BDD contributed data and designed the analysis. BS designed the analysis and wrote the paper.

Funding Open Access funding enabled and organized by Projekt DEAL. No funding was received for this research.

Data availability The data were attached to the manuscript as a supplementary file.

\section{Declartions}

Conflict of interest All authors certify that they have no affiliations with or involvement in any organization or entity with any financial interest (such as honoraria; educational grants; participation in speakers' bureaus; membership, employment, consultancies, stock ownership, or other equity interest; and expert testimony or patent-licensing arrangements), or non-financial interest (such as personal or professional relationships, affiliations, knowledge or beliefs) in the subject matter or materials discussed in this manuscript.

Ethical approval All procedures performed in studies involving human participants were in accordance with the ethical standards of the institutional research committee (Center for Limbal Stem Cell Research and Congenital Aniridia) and with the 1964 Helsinki Declaration and its later amendments or comparable ethical standards. This manuscript did not involve any kind of animal research. All the authors consent to the publication of this manuscript in International Ophthalmology. For this type of study, formal consent is not required.

Informed consent Informed consent was obtained from all individual participants included in the study.

Open Access This article is licensed under a Creative Commons Attribution 4.0 International License, which permits use, sharing, adaptation, distribution and reproduction in any medium or format, as long as you give appropriate credit to the original author(s) and the source, provide a link to the Creative Commons licence, and indicate if changes were made. The images or other third party material in this article are included in the article's Creative Commons licence, unless indicated otherwise in a credit line to the material. If material is not included in the article's Creative Commons licence and your intended use is not permitted by statutory regulation or exceeds the permitted use, you will need to obtain permission directly from the copyright holder. To view a copy of this licence, visit http://creativecommons.org/licenses/by/4.0/.

\section{References}

1. Foster MC (1898) Congenital irideremia. Arch Ophthalmol 27(4):593-615

2. Käsmann-Kellner B, Seitz B (2014) Congenital aniridia or PAX6-Syndrome? Ophthalmologe 111:1144 
3. Käsmann-Kellner B, Viestenz A, Seitz B (2015) Aniridia guides and aniridia syndrome (PAX6 syndrome): Dos and Don'ts in clinical care \& Implementation of supra-regional "Aniridia Guides" can lessen progressive vision loss and improve comprehensive and individualized medical care. In: Parekh M, Poli B, Ferrari S, Teofil C, Ponzi D (eds) Aniridia-recent development in scientific and clinical research. Springer, Switzerland, pp 123-154

4. Lagali N, Wowra B, Fries FN et al (2020) Early phenotypic features of aniridia-associated keratopathy and association with PAX6 coding mutations. Ocul Surf 18(1):130-140

5. Jordan T, Hanson I, Zalatayev D et al (1992) The human PAX6 gene is mutated in two patients with aniridia. Nat Genet 1(5):328-332

6. Akpek EK, Harissi-Dagher M, Petrarca R et al (2007) Outcomes of Boston keratoprosthesis in aniridia: a retrospective multicenter study. Am J Ophthalmol 144:227-231

7. Chen TC, Walton DS (1999) Goniosurgery for prevention of aniridic glaucoma. Arch Ophthalmol 117:1144-1148

8. Seitz B, Käsmann-Kellner B, Viestenz A (2014) Stage-related therapy of congenital aniridia. Ophthalmologe 111:1164-1171

9. Ramaesh K, Ramaesh T, Dutton GN et al (2005) Evolving concepts on the pathogenic mechanisms of aniridia related keratopathy. Int J Biochem Cell Biol 37:547-557

10. Brandt JD, Casuso LA, Budenz DL (2004) Markedly increased central corneal thickness: an unrecognized finding in congenital aniridia. Am J Ophthalmol 137:348-350

11. Lagali N, Wowra B, Fries FN et al (2020) PAX6 mutational status determines aniridia-associated keratopathy phenotype. Ophthalmology 127(2):273-275

12. Seitz B, Das S, Sauer R et al (2011) Simultaneous amniotic membrane patch in high-risk keratoplasty. Cornea 30:269-272

13. Borderie VM, Levy O, Georgeon C et al (2018) Simultaneous penetrating keratoplasty and amniotic membrane transplantation in eyes with a history of limbal stem cell deficiency. J Fr Ophtalmol 4:583-591

14. Lopez-Garcia JS, Rivas L, Garcia-Lozano I et al (2008) Autologous serum eyedrops in treatment of aniridic keratopathy. Ophthalmology 115:262-267

15. Bali S, Filek R, Si F, Hodge W (2016) Systemic immunosuppression in high-risk penetrating keratoplasty: a systematic review. J Clin Med Res 8(4):269-276

16. Chow S, Cook SD, Tole DM (2015) Long-term outcomes of high-risk keratoplasty in patients receiving systemic immunosuppression. Cornea 34(11):1395-1399

17. Yazdanpanah G, Bohm KJ, Hassan OM et al (2020) Management of congenital aniridia-associated keratopathy: long-term outcomes from a tertiary referral center. Am J Ophthalmol 210:8-18

18. Thomasen H, Schroeter J, Reinhard T et al (2018) Good practice procedures for acquisition and preparation of cryopreserved human amniotic membranes from donor placentas. Ophthalmologe 115(10):855-867

19. Wu MF, Stachon T, Seitz B et al (2017) Effect of human autologous serum and fetal bovine serum on human corneal epithelial cell viability, migration and proliferation in vitro. Int J Ophthalmol 10(6):908-913
20. Spaniol K, Koerschgen L, Sander O et al (2014) Comparison of application systems for autologous serum eye drops. Curr Eye Res 39(6):571-579

21. Vicente A, Byström B, Pedrosa Domellöf F (2018) Altered signaling pathways in aniridia-related keratopathy. Invest Ophthalmol Vis Sci 59(13):5531-5541

22. Latta L, Nordström K, Stachon T et al (2019) Expression of retinoic acid signaling components $\mathrm{ADH} 7$ and ALDH1A1 is reduced in aniridia limbal epithelial cells and a siRNA primary cell based aniridia model. Exp Eye Res 179:8-17

23. Rubelowski JM, Latta L, Katiyar P et al (2020) HCE-T cell line lacks cornea-specific differentiation markers compared to primary limbal epithelial cells and differentiated cornea epithelium. Graefes Arch Clin Exp Ophthalmol 258(3):565-575

24. Viestenz A, Seitz B, Deland E et al (2018) Clinical anatomy of the anterior chamber angle in congenital aniridia and consequences for trabeculotomy/cyclophotocoagulation. Clin Anat 31(1):64-67

25. Lee H, KhanO'keefe RM (2008) Aniridia: current pathology and management. Acta Opthalmol 86:708-715

26. Jirsova K, Gary L, Jones A (2017) Amniotic membrane in ophthalmology: properties, preparation, storage and indications for grafting - a review. Cell Tissue Bank 18:193-204

27. Eliason JA, McCulley JP (1990) A comparison between interrupted and continuous suturing techniques in keratoplasty. Cornea 9(1):10-16

28. Seitz B, Resch MD, Schlötzer-Schrehardt U et al (2006) Histopathology and ultrastructure of human corneas after amniotic membrane transplantation. Arch Ophthalmol 142(10): 1487-1490

29. Resch MD, Schlötzer-Schrehardt U, Hofmann-Rummelt C et al (2006) Integration patterns of cryopreserved amniotic membranes into the human cornea. Ophthalmology 113(11):1927-1935

30. Shimpei K, Yoshinori O, Satoshi K et al (2020) Quantitative analysis of the association between follow-up duration and severity of limbal stem cell deficiency or visual acuity in aniridia. Invest Ophthalmol Vis Sci 61(6):57

31. Alfaro Rangel R, Lepper S, Szentmary N et al (2021) Herpes simplex virus keratitis in a university tertiary referral centre-clinical features and surgical approaches. Klin Monbl Augenheilkd Epub: Feb 19

32. Arraquer RI, Pareja-Aricò L, Gómez-Benlloch A et al (2019) Risk factors for graft failure after penetrating keratoplasty. Medicine (Baltimore) 98(17):e15274

33. Bakhtiari P, Chan C, Welder JD et al (2012) Surgical and visual outcomes of the type I Boston Keratoprosthesis for the management of aniridic fibrosis syndrome in congetinal aniridia. Am J Ophthalmol 153(5):967-971

34. Lang S, Böhringer D, Geerling G et al (2017) Long-term results of allogenic penetrating limbo-keratoplasty: 20 years of experience. Eye 31:372-378

Publisher's Note Springer Nature remains neutral with regard to jurisdictional claims in published maps and institutional affiliations. 